International Mathematical Forum, Vol. 8, 2013, no. 2, 67 - 72

\title{
Cubic Residue Characters
}

\author{
Dilek Namli \\ Balikesir Universitesi, Fen-Edebiyat Fakultesi \\ Matematik Bolumu, 10145 Balikesir, Turkey \\ dilekd@balikesir.edu.tr
}

\begin{abstract}
In this study, we investigate for cubic residues of the known results on quadratic residues. We find solutions conditions the equations of cubic residues of the form $x^{3} \equiv a(p)$ and $x^{3} \equiv a(\pi)$.
\end{abstract}

\section{Mathematics Subject Classification: 11A41; 11A15}

Keywords: Rational prime; comlex prime; non-cubic residue

\section{Introduction}

The solutions conditions of linear and quadratic congruence are very well known. In this study we obtain the related to the results.solution conditions of the cubic congruence in D modes prime and rational prime.

\section{Results}

Definition 2.1 If $\pi$, is the prime number in $D$, and if it is $\pi \nsim 1-\omega$ (i.e. $N$ $\pi \neq 3$ ), the cubic character of $\alpha$ in mode $\pi$ will be as follows:

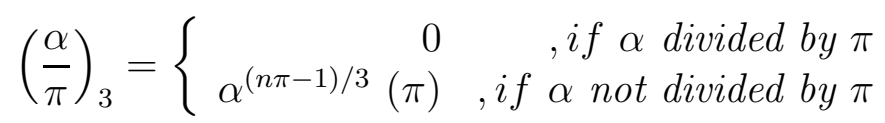

Here, $\alpha^{(n \pi-1) / 3}(\pi)$ is found to be 1 in $\pi$ mode, or it is equal to $\omega$ or $\omega^{2}$. This character functions the role of quadratic residue theory of Legendre symbol in according to the cubic residue theory.

Definition 2.2 If it is $\left(\frac{\alpha}{\pi}\right)_{3}=1, \alpha$ is a cubic residue in the $\pi$ modes. Otherwise, it will be called as non-cubic residue. In the literature, $\chi_{\pi}(\alpha)$ can be replaced with $\left(\frac{\alpha}{\pi}\right)_{3}$. 
Conclusion 2.3 The multiplication of two cubic residues two elements which are non-cubic residues ( $\omega$ and $\omega^{2}$ ) from different types will be a cubic residue. Besides, the multiplication of a cubic residue and a non-cubic residue ( $\omega$ or $\omega^{2}$ ) and two non-cubic residues from the same types ( $\omega$ and $\omega$ or $\omega^{2}$ and $\omega^{2}$ ) will be a non-cubic residue. It is very significant here to know that this case is different from quadratic residues.

Proof. This can be seen in the definition of cubic residue character.?

Theorem 2.4 Suppose that $\pi$ is the prime in $D$ and that it is $N \pi=p$. If the congruence of $x^{3} \equiv a(p)$ can be solved, then the congruence of $x^{3} \equiv a(\pi)$ can also be solved.

Proof. That can be seen in the $p=\pi \bar{\pi}$.

Example 2.5 Let's suppose tat is $\alpha=5+8 \omega$ and $\pi=1+3 \omega$. In that case, it is $N(\alpha)=49$ and $N(\pi)=7$. As it is $7 \mid 49$, as part of description, it will be $\left(\frac{\alpha}{\pi}\right)_{3}=0$. In the reality; it is $\left(\frac{5+8 \omega}{1+3 \omega}\right)_{3}=(5+8 \omega)^{\frac{7-1}{3}}=(5+8 \omega)^{2}$ (7) and as it is $\omega \equiv 2(7)$, the following conguence will be obtained;

$$
(5+8 \omega)^{2} \equiv(5+8.2)^{2} \equiv(21)^{2} \equiv 0(7) .
$$

Theorem 2.6 i) It is $\left(\frac{\alpha \beta}{\pi}\right)_{3}=\left(\frac{\alpha}{\pi}\right)_{3}\left(\frac{\beta}{\pi}\right)_{3}$

ii) If $\alpha \equiv \beta(\pi)$ then is $\left(\frac{\alpha}{\pi}\right)_{3}=\left(\frac{\beta}{\pi}\right)_{3}$.

Proof. i) It will be $\left(\frac{\alpha \beta}{\pi}\right)_{3} \equiv(\alpha \beta)^{(N \pi-1) / 3} \equiv \alpha^{(N \pi-1) / 3} \cdot \beta^{(N \pi-1) / 3} \equiv\left(\frac{\alpha}{\pi}\right)_{3}\left(\frac{\beta}{\pi}\right)_{3}$

ii) If $\alpha \equiv \beta(\pi)$, it will be $\left(\frac{\alpha}{\pi}\right)_{3}=\alpha^{(N \pi-1) / 3} \equiv \beta^{(N \pi-1) / 3} \equiv\left(\frac{\beta}{\pi}\right)_{3}$.

Theorem 2.7 i) $\overline{\left(\frac{\alpha}{\pi}\right)_{3}}=\left(\frac{\alpha}{\pi}\right)_{3}^{2}=\left(\frac{\alpha^{2}}{\pi}\right)_{3}$ and

i) $\overline{\left(\frac{\alpha}{\pi}\right)_{3}}=\left(\frac{\bar{\alpha}}{\pi}\right)_{3}$.

Proof. In the description of cubic residue character, $\left(\frac{\alpha}{\pi}\right)_{3}$ is equal to $1, \omega$ or $\omega^{2}$ and the square of each of these numbers is equal to their conjugate. When we consider that it is $N \bar{\pi}=N \pi$, what we have is i) and ii).

Theorem 2.8 If $\pi$, is the prime number in $D$ and let's suppose that it is $N \pi \neq 3$. Then, it is $\left(\frac{-1}{\pi}\right)_{3}=1$.

Proof. If $\pi$ is prime number, then it is $\mathrm{N} \pi=\mathrm{p}$ providing that $\mathrm{p} \equiv 1$ (3) is a rational prime number. If it is $\mathrm{p} \equiv 1(3)$, and then it is $\mathrm{p}=3 \mathrm{k}+1, \mathrm{k} \in \mathbf{Z}$ and as $\mathrm{p}$ is a prime number, $\mathrm{k}$ will be an even number. Then, as it is

$$
\left(\frac{-1}{\pi}\right)_{3}=(-1)^{(N \pi-1) / 3}
$$


it is $N \pi-1=p-1=3 k+1-1=3 k$ and therefore, it is

$$
\left(\frac{-1}{\pi}\right)_{3}=(-1)^{\frac{3 k}{3}}=(-1)^{k} .
$$

As the $k$ is an even, then it is

$$
\left(\frac{-1}{\pi}\right)_{3}=1
$$

If it is $q \equiv 2(3)$ and $q$ is a rational prime number, then $q$ is a prime number in $D$. As it is $N q=q \cdot \bar{q}=q^{2}$, it is $N q-1=q^{2}-1$. As it is $q \equiv 2(3)$ and also it is a prime number, then $q$ is an odd number. In that case, $N q-1$ is an even number and therefore, $\frac{N q-1}{3}$ is also an even number. If this is the case,it is $\left(\frac{-1}{q}\right)_{3} \equiv(-1)^{(N q-1) / 3}=1$.

Remark 2.9 The cubic character of -1 in each $\pi$ mode, will be 1 can be seen from that is $(-1)^{3}=-1$. We know that $p \equiv 1(3)$ as a prime number and $N \pi=p$ and $a^{\frac{p-1}{3}} \equiv 1, \omega, \omega^{2}(p)$. In other words, the $\frac{p-1}{3}$. powers of the elements of $\mathbb{Z}_{p}-\{0\}$ are $1, \omega, \omega^{2}$ which are equivalent to the elements in $\mathbb{Z}_{p}$. Therefore, the element of $p-1$ are some how gathered under 3 groups. In each of these groups, the number of elements is $\frac{p-1}{3}$.

$K_{p}=\left\{k \mid k\right.$, is a residue $\frac{p-1}{3}$ th.a different from zero in $p$ mode $\}$ which can be considered to be as a main description. In other words, the $K_{p}$, the powers of $\frac{p-1}{3} t h$. of the elements of $\mathbb{Z}_{p}-\{0\}$ consist of values in $p$ mode.

Theorem 2.10 $K_{p}$, is a group depending on the multiplication in $\mathbb{Z}_{p}$ and in fact it is a subgroup of $\mathbb{Z}_{p}^{*}$.

Proof. We have the following as $K_{p}=\left\{1, \omega, \omega^{2}\right\}$,

i) We see that it is $a(b c)=(a b) c$ for $\forall a, b, c \in K_{p}$.

ii) 1 is the unit element of $K_{p}$.

iii) As it is $1.1=1, x \cdot \omega^{2}=1$, the opposite of 1 is 1 , the opposite of $\omega$ is $\omega^{2}$ and the opposite of $\omega^{2}$ is $\omega$.

$K_{p}$ from i, ii and iii is a group under the multiplication.

Now let's see that the $\forall a, b \in K_{p}$ is $a b^{-1} \in \mathbb{Z}_{p}^{*}$.

$1 . \omega^{-1}=1 . \omega^{2}=\omega^{2} \in \mathbb{Z}_{p}^{*}, 1 .\left(\omega^{2}\right)^{-1}=1 . \omega=\omega \in \mathbb{Z}_{p}^{*}, \omega \cdot \omega^{-1}=\omega \cdot \omega^{2} \equiv 1 \in \mathbb{Z}_{p}^{*}$, $\omega \cdot\left(\omega^{2}\right)^{-1}=\omega \cdot \omega=\omega^{2} \in Z_{p}^{*}, 1 \cdot 1^{-1}=1.1 .=1 \in Z_{p}^{*}$ and $\omega^{2} \cdot\left(\omega^{2}\right)^{-1}=\omega^{3} \in$ $Z_{p}^{*}$.

Example 2.11 Let's the $K_{7}$ and $K_{13}$. It is $p=7$ and $\frac{p-1}{3}=2$. In mode 7 , it is $1^{2} \equiv 1,2^{2} \equiv 4,3^{2} \equiv 2,4^{2} \equiv 2,5^{2} \equiv 4,6^{2} \equiv 1$ and it is $\omega \equiv 4(7), \omega^{2} \equiv 2(7)$, 
it is also $K_{7}=\{1,2,4\} \equiv\left\{1, \omega, \omega^{2}\right\}$. Now, let's suppose that $p=13$. Then it is $\frac{p-1}{3}=4$. In mode 13 , as it is $1^{4} \equiv 1,2^{4} \equiv 3,3^{4} \equiv 3,4^{4} \equiv 9,5^{4} \equiv 1,6^{4} \equiv$ $9,7^{4} \equiv 9,8^{4} \equiv 1,9^{4} \equiv 9,10^{4} \equiv 3,11^{4} \equiv 3,12^{4} \equiv 1$ and $\omega \equiv 9(13), \omega^{2} \equiv 3(13)$, what we have is $K_{13}=\{1,3,9\} \equiv\left\{1, \omega, \omega^{2}\right\}$.

Theorem 2.12 (Cubic reciprocity law) Let $\pi_{1}$ and $\pi_{2}$ is 1.type prime, that $N \pi_{1}, N \pi_{2} \neq 3$ and $N \pi_{1} \neq N \pi_{2}$. Then is $\left(\frac{\pi_{1}}{\pi_{2}}\right)_{3}=\left(\frac{\pi_{2}}{\pi_{1}}\right)_{3}$ [5].

Theorem 2.13 If it is $\pi=a+b \omega$ and $\pi \equiv 2(3)$ then we have $\left(\frac{\omega}{\pi}\right)_{3}=\omega^{(a+b+1) / 3}$ [4]

Theorem 2.14 If it is $\pi=a+b \omega$ and $\pi \equiv 2(3)$ then it is $\left(\frac{1-\omega}{\pi}\right)_{3}=\omega^{2(a+1) / 3}$ [4].

Theorem 2.15 If $\pi$, is a 1.type rational prime number, then it is $\left(\frac{2}{\pi}\right)_{3}=1$. In other words, 2 is a cubic residue in every $q$ mode providing that $\pi=q>2$ it is 1.type rational prime number.

Proof. Suppose that $\pi=q$ is a rational prime number. It cannot be $q=2$, because then it is $2 \mid 2$ and $\left(\frac{2}{q}\right)_{3}=0$. While $q \equiv 2(3)$ is a rational prime number, we know that in mode $q$, there are $q$ pieces of cubic residue, in other words, in $q$ mode, each a number is a cubic residue. Therefore, 2 in mode $q$ is a cubic residue.

Theorem 2.16 If it is $\pi=a+b \omega, 1$.type complex prime number, to solve the $x^{3} \equiv 2(\pi)$ the necessary and sufficient condition is $\pi \equiv 1(2)$, in other words it needs to be $a \equiv 1$ (2) and $b \equiv 0$ (2) .

Proof. Suppose that $x^{3} \equiv 2(\pi)$ is something which can be solved. Then, it is $\left(\frac{2}{\pi}\right)_{3}=1$. As both of 2 and $\pi$ are 1.type prime numbers, as required by the cubic reciprocity law, we can write as follows: $\left(\frac{2}{\pi}\right)_{3}=\left(\frac{\pi}{2}\right)_{3}$. As it is $\left(\frac{\pi}{2}\right)_{3} \equiv \pi^{(N 2-1) / 3}(2)$ and $N(2)=2^{2}=4$, it is $\left(\frac{\pi}{2}\right)_{3} \equiv \pi(2)$. Therefore, it needs to be $\left(\frac{\pi}{2}\right)_{3} \equiv \pi \equiv 1(2)$ so that we can have the following $;\left(\frac{\pi}{2}\right)_{3}=1$. The reverse case can also be possible.

Example 2.17 Can the following congruence is a soluble one?

$$
x^{3} \equiv 2(5+6 \omega)
$$

As $\pi=5+6 \omega$ is 1.type, in other words, $\pi \equiv 2$ (3) and it is $\pi \equiv 1$ (2), as required by the theorem 16 , it is $\left(\frac{2}{5+6 \omega}\right)_{3}=1$. In other words, the congruence of $x^{3} \equiv 2(5+6 \omega)$ can be solved. By using the Theorem 2.15, it is

$$
\begin{aligned}
\left(\frac{2}{5+6 \omega}\right)_{3} & =\left(\frac{5+6 \omega}{2}\right)_{3}=(5+6 \omega)^{N(2)-1}=5+6 \omega \\
& \equiv 1+0 \omega(2) \\
& \equiv 1(2) .
\end{aligned}
$$


Remark 2.18 Gauss, if it is $p \equiv 1(3)$, that demonstrates that $A$ and $B$ whole numbers exist as in $4 p=A^{2}+27 B^{2}$ and that these $A$ and $B$ whole numbers can be determined with only one single way except for signs.

Theorem 2.19 Suppose that it is $\pi=a+b \omega, 1$.type prime number and $N \pi=$ $p=a^{2}-a b+b^{2}$. If it is $p \equiv 1(3)$, to be able to solve the congruence of $x^{3} \equiv 2$ $(p)$ the necessary and sufficient condition is to find the $C$ and $D$ whole integers to make it $p=C^{2}+27 D^{2}$.

Proof. If the congruence of $x^{3} \equiv 2(p)$ can be solved, then the congruence of $x^{3} \equiv 2(\pi)$ can also be solved and as required by the Theorem 2.15 , it is $\pi \equiv 1$ (2). If it is $p=a^{2}-a b+b^{2}$ then it is $4 p=4 a^{2}-4 a b+4 b^{2}=(2 a-b)^{2}+3 b^{2}$. Here if it is $2 a-b=A, \frac{b}{3}=B$, as $A$ is an odd number and $b$ is an even number, $A$ and $B$ are even numbers. Then, can be written as $D=\frac{B}{2}$ and $C=\frac{A}{2}$ and thus obtained $p=C^{2}+27 D^{2}$.

Now let's suppose that there exist $\mathrm{C}$ and $\mathrm{D}$ whole integers to make the $p=C^{2}+27 D^{2}$, then it is $4 p=(2 C)^{2}+27(2 D)^{2}$. With this equality, is obtained $B=\mp 2 D$. In other words, $B$, and $b$ are even numbers. If sothe following equality is obtained $\pi=a+b \omega \equiv 1$ (2) (but the following cannot be obtained $a \equiv 0(2)$, because, if so, it is $\pi \equiv 0(2))$ and the results is seen from the Theorem 2.15 .

Example 2.20 Let's take $p=19$. The number $p$ cannot be written as $C^{2}+$ $27 D^{2}$, the congruence of $x^{3} \equiv 2$ (19) cannot be solved. In fact, as it is; $\left(\frac{2}{19}\right)_{3}=$ $2^{N(19)-1 / 3}=2^{120} \equiv 11(19)$ and $\omega \equiv 11$ (19), the following is obtained; is obtained $\left(\frac{2}{19}\right)_{3} \equiv \omega(19)$. Now, let's take $\pi=5+3 \omega, 1$. type prime number in which it is $N \pi=19$.

$$
\left(\frac{2}{5+3 \omega}\right)_{3}=\left(\frac{5+3 \omega}{2}\right)_{3}=(5+3 \omega)^{N(2)-1 / 3}=5+3 \omega \equiv 1+\omega
$$

and

$$
\begin{gathered}
1+\omega=-\omega^{2} \equiv(-1) \cdot \omega^{2} \\
\equiv 1 . \omega^{2}(2)
\end{gathered}
$$

As it is, the following congruence is obtained

$$
\left(\frac{2}{5+3 \omega}\right)_{3}=\omega^{2}
$$

and therefore, the congruence of $x^{3} \equiv 2(5+3 \omega)$ cannot be solved. 
On the other hand, as the number of $p=31$ can be written as $2^{2}+27.1=31$, in reality, as it is $\left(\frac{2}{31}\right)_{3}=2^{N(31)-1 / 3}=2^{320} \equiv 1(31)$, the congruence of $x^{3} \equiv 2$ (31) can be solved and it is easy to see that $x=4$. With the help of the other roots can be found as $x \omega \equiv 20$ and $x \omega^{2} \equiv 7(31)$.

Let's take now the $\pi=5+6 \omega$ 1.type prime number which is $N \pi=31$.

$$
\left(\frac{2}{5+6 \omega}\right)_{3}=\left(\frac{5+6 \omega}{2}\right)_{3}=(5+6 \omega)^{N(2)-1 / 3}=5+6 \omega \equiv 1
$$

is obtained and thus 2 is found to be a cubic residue in $5+6 \omega$ mode.

When $p \equiv 1(3)$, as $\omega \in \mathbb{Z}_{p}$, we are more interested in the cubic residues in $p$ mode rather than the residues in $\pi=a+b \omega$ prime mode in $D$. Considering that $k>1$ is a whole integer, as it is $p=3 k$ and $p \equiv 2(3)$, there is no $\pi=a+b \omega$ prime number whose in $D$ norm is $p$ norm, and as definition of cubic residue concept is described when it is $N \pi \neq 3$, there will be no limitations.

\section{References}

[1] Jones, G.A., Jones J.M., Elemantary Number Theory, Springer-Verlag, Newyork , (1998), S.37-140.

[2] Flath, D.E., Introduction to Number Theory, A.Wiley-Interscience Publication, (1989), s.63-104.

[3] Leveque, W.J., Fundamentals of Number Theory, Dover Publications, Newyork, (1997), s.47-93, 97-120, 270-273.

[4] Stark, H.M., An Introduction to Number Theory, Cambridge, London, (1979), s.51-117.

[5] Sun, Z.H., "On the theory of cubic residues and nonresidues", Acta Arithmetica J., 4 (1998), s.291-335.

\section{Received: September, 2012}

